\title{
Effectiveness of endosponge therapy for the management of presacral abscesses following rectal surgery
}

\author{
J. F. Huisman ${ }^{1}$ - H. L. van Westreenen ${ }^{2}$ - E. J. van der Wouden ${ }^{1}$. H. F. A. Vasen ${ }^{3}$. E. J. R. de Graaf ${ }^{4}$. \\ P. G. Doornebosch ${ }^{4} \cdot$ T. J. Tang ${ }^{5} \cdot$ I. Schot ${ }^{5} \cdot$ R. M. Brohet ${ }^{6} \cdot$ W. H. de Vos tot Nederveen Cappel ${ }^{1} \cdot$ M. Vermaas $^{4}$
}

Received: 8 January 2019 / Accepted: 13 May 2019 / Published online: 23 July 2019

(c) Springer Nature Switzerland AG 2019

\begin{abstract}
Background Anastomotic leak after rectal surgery is reported in 9\% (range 3-28\%) of patients. The aim of our study was to evaluate the effectiveness of endosponge therapy for anastomotic. Endpoints were the rate of restored continuity and the functional bowel outcome after anastomotic leakage.

Methods This was a multicenter retrospective observational cohort study. All patients with symptomatic anastomotic leakage after rectal surgery who had endosponge therapy between January 2012 and August 2017 were included. Functional bowel outcome was measured using the low anterior resection syndrome (LARS) score system.

Results Twenty patients were included. Eighteen patients had low anterior resection (90\%) for rectal cancer. A diverting ileostomy was performed at primary surgical intervention in 14 patients $(70 \%)$. Fourteen patients $(70 \%)$ were treated with neoadjuvant (chemo-)radiotherapy. The median time between primary surgical intervention and first endosponge placement was 21 (5-537) days. The median number of endosponge changes was 9 (2-28). The success rate of the endosponge treatment was $88 \%$ and the restored gastrointestinal continuity rate was $73 \%$. A chronic sinus occurred in three patients (15\%). All patients developed LARS, of which 77\% reported major LARS.

Conclusions Endosponge therapy is an effective treatment for the closure of presacral cavities with high success rate and leading to restored gastrointestinal continuity in $73 \%$. However, despite endosponge therapy many patients develop major LARS.
\end{abstract}

Keywords Rectal neoplasms $\cdot$ Anastomotic leak $\cdot$ Endosponge $\cdot$ Patient reported outcome measures $\cdot$ LARS score

\section{Introduction}

Anastomotic leakage (AL) after rectal surgery is reported in $9 \%$ (range $3-28 \%$ ) of the patients and is associated with forming of presacral abscesses, emergency surgery, morbidity, permanent colostomy, prolonged hospital stay and even mortality [1]. Several risk factors have been associated with A; level of the anastomosis, neoadjuvant (chemo)-radiotherapy (CRT), male gender, tumor size and other comorbidities [2-4]. A recent study reported 30-day postoperative AL rate of $13.4 \%$ in patients with rectal cancer surgery, which increased during follow-up to $20 \%$ [5].

A significant proportion (36\%) of patients with $\mathrm{AL}$ develop a chronic sinus, $50 \%$ of which may heal spontaneously over time $[5,6]$. Conventional treatment consists of antibiotics, radiological therapy (transanal or transgluteal drainage) or surgical therapy (diverting loop ileostomy, endoluminal drainage or dismantling of the anastomosis).

5 Department of Gastroenterology and Hepatology, IJsselland Hospital, Capelle aan de IJssel, The Netherlands

6 Department of Epidemiology and Statistics, Isala, Zwolle, The Netherlands 
Endosponge therapy is a relatively novel minimally invasive endoluminal vacuum therapy for presacral abscesses that aims to clean the presacral cavity that subsequently collapses [7-10]. Therefore, it may prevent the development of a chronic sinus and may improve the anastomotic healing rate. A review by Strangio et al. reported high success $(94 \%)$ of endosponge therapy for the treatment of presacral abscesses [11]. Gardenbroek et al. reported high effectiveness of vacuum-assisted early transanal closure of $\mathrm{AL}$ in patients with inflammatory bowel disease (IBD) who had ileal pouch-anal anastomosis (IPAA) [12]. Borstlap et al. recently demonstrated restored gastrointestinal continuity in $67 \%$ of the patients who had vacuum-assisted early transanal closure after AL, especially when the endosponge therapy was started within 3 weeks of primary surgical intervention [13]. The aim of our study was to evaluate the effectiveness of endosponge therapy. Endpoints were the rate of restored continuity and the functional bowel outcome in patients with anastomotic leakage after rectal surgery.

\section{Materials and methods}

\section{Study design and patient selection}

This retrospective cohort study was performed in two Dutch high-volume colorectal cancer centers: Isala hospital and IJsselland hospital. All eligible patients with symptomatic AL after rectal surgery treated with endosponge therapy between January 2012 and August 2017 were included. Patients with postoperative signs of AL and AL confirmed by computed tomography (CT) scan were considered eligible. Patients with colonic cancer, patients who underwent Hartmann's procedure as primary surgical procedure and patients who underwent transanal endoscopic microsurgery (TEM) were excluded. The study was approved by the medical ethics committee of Isala hospital (reference number: 171215).

All patients were discussed in a preoperative multidisciplinary team meeting and had an open or laparoscopic total mesorectal excision (TME) or IPAA. In case of surgery for rectal cancer, neoadjuvant short-course radiotherapy $(5 \times 5 \mathrm{~Gy}$.) or long-course chemo-radiotherapy was given depending on the stage of disease. The presacral abscess was evaluated during every endosponge exchange. The abscess was considered closed if the cavity was covered with granulation tissue and the size of the cavity was too small for another endosponge placement. At that moment, the endosponge therapy stopped.

\section{Endosponge procedure}

The endosponge therapy was performed with or without conscious sedation depending on the patient's preference. Depending on the size of the cavity 1-3 polyurethane endosponges (Endo-SPONGE ${ }^{\circledR}$, B. Braun Medical B.V., Melsungen, Germany) were placed in the deepest point of the presacral cavity through a plastic overtube under endoscopic guidance. If necessary, the endosponge was tapered to achieve collapse of the cavity. The endosponge was connected to a vacuum suction device that created a constant negative pressure of $150 \mathrm{mmHg}$. The endosponges were changed twice a week to prevent the granulation tissue from growing into the endosponge. In most patients, the first endosponge was placed by the surgeon and gastroenterologist together. If needed, the anastomotic defect was dilated with an endoscopic balloon to facilitate drainage and the placement of the endosponge which if necessary was done under radiologic assistance. The next endosponge changes were performed by the gastroenterologist alone. Depending on surgeon preference, transanal closure of the defect was performed after a short period of endosponge therapy (vacuum-assisted early transanal closure) to achieve shorter endosponge therapy duration. A detailed description of this procedure can be found in the paper of Borstlap et al. [13].

\section{Outcomes}

Primary outcome was the restored gastrointestinal continuity rate at the end of the follow-up.

Secondary outcomes were the success rate of the endosponge therapy, presence of a chronic sinus and the functional bowel outcome after AL. Success of the endosponge treatment was defined as a cavity reduced in size and covered with granulation tissue that was too small to allow placement of a new endosponge at the end of the endosponge therapy. A chronic sinus was defined as a proven presacral abscess that was still present 1 year after primary surgical intervention.

\section{Functional bowel outcome}

Functional bowel outcome was assessed postoperatively using the validated quality of life questionnaire (low anterior resection syndrome score (LARS score)) [14, 15]. The results of the LARS score were categorized into three groups: (1) no LARS (0-20 points), (2) minor LARS (21-30 points) or (3) major LARS (31-42 points).

A control group was created using the institutional colorectal cancer database, consisting of patients with rectal 
cancer without AL. These patients were matched with the endosponge group for CRT.

\section{Statistical analysis}

All analyses were performed using Statistical Package of Social Sciences version 22 (SPSS). A $p$ value $<0.05$ was considered significant. Normality was tested using Kolmogorov-Smirnov test. Normally distributed variables were described as mean with standard deviation (StD) and nonparametrical distributed variables were described as median with range. Categorical data were tested using Chi-square test or Fisher's exact test. Normally distributed continuous data were tested using Student's $T$ test. Non-parametrical continuous data were tested using Mann-Whitney $U$ test. Additionally, the non-parametrical continuous data were divided into two groups based on the median and tested with Chi-square or Fisher's exact test. Survival analysis was performed to estimate the probability for stoma reversal and success rate of the endosponge after resection using Kaplan-Meier analysis. Start of follow-up was primary resection and end of follow-up was date of interest; stoma reversal date, last endosponge exchange date, date of death or end of follow-up. End of follow-up for patients without stoma reversal or not censored was last hospital visit.

For subgroup analysis, patients were divided into early and late endosponge groups based on the median number of days between primary surgical intervention and start of the endosponge therapy. Patients who started endosponge therapy before the median cutoff point were allocated to the early endosponge group and patients who started endosponge therapy on or after the cutoff point were allocated to the late endosponge group. The difference in stoma reversal between early and late endosponge groups was calculated using the log rank test.

A multivariate linear regression analysis was performed to determine whether occurrence of AL influences the LARS scores taking into account other potential confounders or patient characteristics at baseline.

\section{Results}

\section{Baseline characteristics}

A total of 20 patients were eligible for inclusion in our study. Baseline characteristics are presented in Table 1. Fourteen of $20(70 \%)$ patients were diverted during primary surgery. Three of the six patients who were not initially diverted at primary surgical intervention were diverted at the time AL was detected, two patients received endosponge therapy without diverting ileostomy and the other patient had Hartmann's procedure followed by endosponge therapy of the presacral abscess. Reintervention before the start of the endosponge therapy was performed in 8 of 20 patients: surgical drain placement $(n=4)$, diverting ileostomy $(n=3)$ and dismantling of the anastomosis with Hartmann's procedure $(n=1)$. In 3 of the 20 enrolled patients, the anastomotic defect was transanally closed after a median of $2[2$, $3]$ endosponge changes.

The median time between primary surgical intervention and anastomotic leak detection, median time between primary surgical intervention and first endosponge placement, median number of endosponge changes and median duration of the endosponge therapy are presented in Table 2. No endosponge-related adverse events were reported.

Table 1 Baseline characteristics

\begin{tabular}{|c|c|c|c|c|}
\hline & Total patients & Early endosponge & Late endosponge & $p$ value \\
\hline$n$ & 20 & 10 & 10 & \\
\hline Male $^{\mathrm{a}}$ & $14(70)$ & $5(50)$ & $9(90)$ & $0.14^{\mathrm{c}}$ \\
\hline Age (years) ${ }^{b}$ & $64 \pm 10$ & $64 \pm 11$ & $64 \pm 10$ & $0.95^{\mathrm{e}}$ \\
\hline Distance $(\mathrm{cm})$ from anal verge ${ }^{\mathrm{b}}$ & $8.5[5-12]$ & $8[6-12]$ & $9[5-10]$ & $0.27^{\mathrm{d}}$ \\
\hline Etiology ${ }^{\mathrm{a}}$ & & & & $0.47^{\mathrm{c}}$ \\
\hline Rectal cancer & $18(90)$ & $8(80)$ & $10(100)$ & \\
\hline Inflammatory bowel disease & $2(10)$ & $2(20)$ & - & \\
\hline Type of procedure ${ }^{a}$ & & & & $0.47^{\mathrm{c}}$ \\
\hline Total mesorectal excision & $18(90)$ & $8(80)$ & $10(100)$ & \\
\hline Ileal pouch-anal anastomosis & $2(10)$ & $2(20)$ & - & \\
\hline Laparoscopic procedure $^{\mathrm{a}}$ & $16(80)$ & $9(90)$ & $7(70)$ & $0.58^{\mathrm{c}}$ \\
\hline Neoadjuvant chemo-radiotherapy ${ }^{a}$ & $14(70)$ & $5(50)$ & $9(90)$ & $0.14^{\mathrm{c}}$ \\
\hline Short-course neoadjuvant therapy & $11(55)$ & $4(40)$ & $7(70)$ & \\
\hline Long-course neoadjuvant therapy & $3(15)$ & $1(10)$ & $2(20)$ & \\
\hline
\end{tabular}

${ }^{\mathrm{a}}$ Count (\%), ${ }^{\mathrm{b}}$ Median [range], ${ }^{\mathrm{c}}$ Fischer's exact test, ${ }^{\mathrm{d}}$ Mann-Whitney $U$ test, ${ }^{\mathrm{e}}$ Student's $t$ test 
Table 2 Clinical outcome of endosponge treatment

\begin{tabular}{lllll}
\hline & Total group & Early endosponge & Late endosponge & $p$ value \\
\hline Days until anastomotic leak detection $^{\mathrm{b}}$ & $12[3-67]$ & $10[3-19]$ & $21[4-67]$ & $0.10^{\mathrm{c}}$ \\
Days until first endosponge $^{\mathrm{b}}$ & $21[5-537]$ & $11[5-20]$ & $30[21-537]$ & ${<0.001^{\mathrm{c}}}^{\mathrm{c}}$ \\
Endosponge changes $^{\mathrm{b}}$ & $9[2-28]$ & $6[2-28]$ & $14[2-26]$ & $0.45^{\mathrm{c}}$ \\
Duration endosponge therapy (days) $^{\mathrm{b}}$ & $25[3-115]$ & $20[3-115]$ & $25[5-80]$ & $0.79^{\mathrm{c}}$ \\
Median follow-up (months) $^{\mathrm{b}}$ & $10[3-84]$ & $8[3-25]$ & $12[6-84]$ & $0.08^{\mathrm{c}}$ \\
Success endosponge therapy $^{\mathrm{a}}$ & $17(85)$ & $8(80)$ & $9(90)$ & - \\
Restored continuity $^{\mathrm{a}}$ & $14(70)$ & $7(70)$ & $7(70)$ & - \\
Time until stoma reversal $^{\mathrm{b}}$ & $10[3-15]$ & $7[3-11]$ & $10[6-15]$ & $0.15^{\mathrm{c}}$ \\
Chronic sinus $^{\mathrm{a}}$ & $3(15)$ & $2(20)$ & $1(10)$ & - \\
\hline
\end{tabular}

${ }^{\mathrm{a}}$ Count (\%), ${ }^{\mathrm{b}}$ Median [range], ${ }^{\mathrm{c}}$ Mann-Whitney $U$

\section{Outcome of endosponge therapy}

Endosponge treatment was successful in 17 of 20 patients $(85 \%)$. In 14 of the 20 patients $(70 \%)$, continuity was restored. Six patients received a definitive stoma because of a chronic sinus $(n=3)$, proctectomy $(n=1)$, local recurrence $(n=1)$ and one patient died because of tumor progression. A chronic sinus has developed in three patients (15\%). The median time from primary resection to stoma reversal was 10 [3-15] months (Table 2). The overall cumulative probability of endosponge therapy success was $88 \%$ (95\% CI $=57-97 \%)$ and the overall cumulative probability of stoma removal was $73 \%(95 \% \mathrm{CI}=44-87 \%)$. Individual follow-up characteristics after primary resection are presented in Fig. 1.

\section{Outcome of early versus late endosponge group}

Ten patients were classified as being in an early endosponge group and 10 patients in a late endosponge group based on the study median of 21 days from primary surgery until first endosponge placement. Reasons for the delay of endosponge placement in the late endosponge group were: the presacral abscess became clinical relevant only after 3 weeks $(n=5)$, re-laparotomy with surgical drainage $(n=2)$, end colostomy $(n=1)$, diverting ileostomy $(n=1)$ and antibiotic therapy prior to endosponge therapy $(n=1)$. An overview of the results of the early and late endosponge therapy is presented in Table 2.

In the early group, the endosponge therapy was successful in 8/10 patients and anastomotic healing occurred in $7 / 10$ patients. Two patients had a permanent colostomy because of a chronic sinus, unresolved by endosponge. The other patient without restored gastrointestinal continuity died 6 months after endosponge therapy because of tumor progression. The median time until stoma reversal was 7 months. In the late endosponge group, the endosponge therapy was successful in 9/10 patients and anastomotic healing occurred in 7/10 patients. Three patients did not have intestinal continuity restored because of: chronic sinus

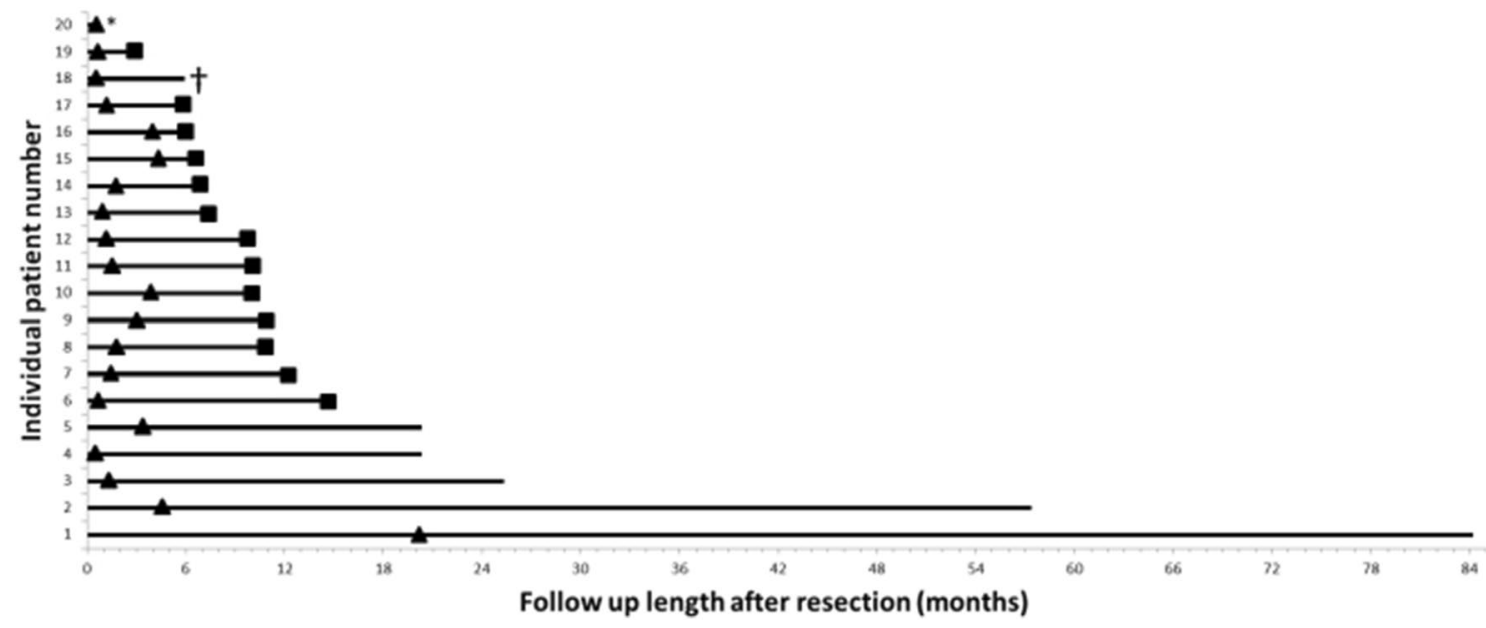

Fig. 1 Individual follow-up characteristics after resection. Fourteen patients (70\%) were reversed successfully. *No primary diverting stoma. Filled triangle: end of endosponge and determining success rate of endosponge. 'Deceased 
$(n=1)$, local recurrence $(n=1)$ and proctectomy $(n=1)$. The patient with a permanent colostomy due to a chronic sinus was treated in the beginning with conventional therapy and has eventually started endosponge therapy 537 days after primary surgical resection without success. The median time until stoma reversal in the late endosponge group was 10 months.

The overall cumulative probability of stoma removal for patients in the early endosponge group was 77\% (95\% $\mathrm{CI}=22-93 \%)$ compared with $70 \%$ (95\% $\mathrm{CI}=23-88 \%)$ for patients in the late endosponge group. This difference in absolute risks was not statistically significant $(p=0.31)$. Also, no statistically significant difference in the success rate of endosponge therapy and the presence of a chronic sinus was found between the early and late endosponge groups.

\section{Quality of life}

Fourteen patients who had endosponge treatment received the questionnaire. Six patients were not invited to fill in the questionnaire because of a permanent colostomy. Thirteen patients $(93 \%)$ responded to the questionnaire. Thirty-two patients in the control group (without $\mathrm{AL}$ ) received the questionnaire. Twenty-one (66\%) of them responded to the questionnaire. Baseline characteristics between the endosponge group and the control group are presented in Table 3.

The median LARS score in the endosponge group was 37 (23-42) points and 30 (4-41) points in the control group $(p=0.009)$. The median time between stoma reversal and date of the quality of life questionnaire was $2.6(0.8-3.5)$ years in the endosponge group and $2.3(1.8-2.8)$ years in the control group $(p=0.47)$. No significant difference between these two groups in age, response to questionnaire time, distance to anal verge, CRT and laparoscopy procedure was found. In the endosponge group, three patients (23\%) had minor LARS and ten patients (77\%) had major LARS. In the control group, five patients (24\%) had no LARS, six patients (29\%) had minor LARS and ten patients (48\%) had major LARS. In the multivariate analysis, the LARS score was significantly associated with endosponge therapy $(\beta=-7.595$, $p=0.02$ ) (Table 3). No significant difference in LARS scores was found between the early and late endosponge groups $(p=0.72)$.

\section{Discussion}

We reported a large series of 20 patients who were treated with endosponge therapy after AL. This study is, to the best of our knowledge, the first study that describes the longterm functional bowel outcome after endosponge therapy in patients with AL compared with a control group consisting of patients without AL after TME. The majority of our patients $(90 \%)$ were treated for rectal cancer and $70 \%$ of the patients received neoadjuvant (chemo-)radiotherapy. There was a high success rate $(88 \%)$ of endosponge therapy, restored bowel continuity rate of $73 \%$ and major LARS in $77 \%$ of the patients after endosponge therapy.

These findings are in line with previous studies that reported on the outcome of endosponge therapy. Mussetto et al. reported successful endosponge therapy with restored gastrointestinal continuity in 10 of the 11 patients (91\%) after anastomotic leakage [16]. Strangio et al. described a completely healed cavity after endosponge therapy in $94 \%$ of the cases and anastomotic healing in 56-92\% of the cases [11]. The majority (90\%) of these patients were treated for rectal cancer and patients with generalized peritonitis were excluded. Borstlap et al. demonstrated an anastomotic healing rate of $67 \%$ in patients with AL who had vacuumassisted early transanal closure [13]. However, $93 \%$ of the
Table 3 Baseline characteristics of patients who developed LARS

\begin{tabular}{llll}
\hline & $\begin{array}{l}\text { Endosponge after anas- } \\
\text { tomotic leakage }\end{array}$ & No anastomotic leakage & $p$ value \\
\hline LARS score $^{\mathrm{b}}$ & $37[23-42]$ & $30[4-41]$ & $0.009^{\mathrm{d}}$ \\
Major LARS $^{\mathrm{a}}$ & $10(77)$ & $10(48)$ & \\
Minor LARS $^{\mathrm{a}}$ & $3(23)$ & $6(29)$ & \\
No LARS $^{\mathrm{a}}$ & - & $5(24)$ & $0.43^{\mathrm{e}}$ \\
Age (years) $^{\mathrm{b}}$ & $67 \pm 7$ & $64 \pm 8$ & $0.26^{\mathrm{d}}$ \\
Response time questionnaire (years) $^{\mathrm{b}}$ & $3.1[1.5-4.5]$ & $2.6[2.3-2.8]$ & $0.46^{\mathrm{d}}$ \\
Time from stoma reversal to question- $^{\mathrm{d}}$ & $2.6[0.8-3.5]$ & $2.3[1.8-2.8]$ & \\
naire date (years) & & $10[5-15]$ & $0.07^{\mathrm{d}}$ \\
Distance tumor to anal verge (cm) $^{\mathrm{b}}$ & $8.5[5-12]$ & $16(76)$ & $0.36^{\mathrm{c}}$ \\
Neoadjuvant chemo-radiotherapy $^{\mathrm{a}}$ & $8(62)$ & $19(91)$ & $0.35^{\mathrm{c}}$ \\
\hline Laparoscopic procedure $^{\mathrm{a}}$ & $10(77)$ & & \\
\hline
\end{tabular}

LARS low anterior resection syndrome

${ }^{\mathrm{a}}$ Count (\%), ${ }^{\mathrm{b}}$ Median [range], ${ }^{\mathrm{c}}$ Fischer's exact test, ${ }^{\mathrm{d}}$ Mann-Whitney $U$ test, ${ }^{\mathrm{e}}$ Student's $t$ test 
patients had persistent AL 2 weeks after transanal closure, requiring conservative monthly endoscopic follow-up (43\%), redo endosponge therapy (32\%), redo transanal surgical closure $(18 \%)$, percutaneous drainage $(4 \%)$, or end colostomy (4\%). Furthermore, they reported that early start of endosponge therapy (before 3 weeks) resulted in higher stoma reversal rate and lower proportion of a chronic sinus. Therefore, early treatment of the presacral abscess might be advocated to improve anastomotic healing rates and reduce the development of a chronic sinus. Our study demonstrated a similar success rate of the endosponge therapy, similar anastomotic healing rates and a lower proportion of a chronic sinus compared to Borstlap et al. [13]. We found no significant difference in our study between the early and late endosponge groups for these parameters.

A chronic sinus occurred in $15 \%$ of the patients in our study. Previous studies reported a persistent sinus rate at 1 year of $48 \%$ after AL without endosponge therapy $[5,6]$. The proportion of chronic sinus found in our study is low compared to these studies, suggesting that endosponge is a good therapy to prevent the development of chronic sinus. However, endosponge was not used in these two earlier studies. Furthermore, a study reported a late abscess in $25 \%$ of the patients after successful endosponge. A prolonged interval between primary surgery and AL detection was the only predictive factor in this study [17]. Until now, no late abscesses were seen in our study.

$\mathrm{AL}$ and chronic sinus are associated with impaired functional bowel outcome. Improvements of the functional bowel outcome can be expected especially in the first year after surgery. In the following years, the functional outcome will remain stable [18]. We assessed the functional outcome using the LARS score questionnaire after a median of more than 2 years after stoma reversal. Patients with AL who had endosponge therapy had significantly higher LARS scores than patients without AL in the control group. We used patients without AL as a control group, because most patients who presented with AL that did not have endosponge therapy had their anastomosis taken down or resected and received an end colostomy.

All patients in our study had LARS (77\% had major LARS and 23\% minor LARS) after endosponge therapy compared to $76 \%$ in the control group. Borstlap et al. also reported high LARS scores after combined surgical and endoscopic treatment for AL ( $81 \%$ had major LARS and $13 \%$ minor LARS) [13]. A systematic review of patients without AL following rectal cancer surgery reported major LARS in $38-62 \%$, minor LARS in $22-28 \%$ and no LARS in $10-38 \%$ [19].

Our study and the results from the previous literature mentioned above demonstrate that patients with AL who had endosponge therapy have a higher risk of developing (severe) LARS compared with patients without AL. The main causes of LARS are probably the AL and fibrosis in the abscess cavity during endosponge therapy causing reduced neorectal compliance. Another explanation of our high LARS scores after endosponge therapy could be that the endosponge group has a lower anastomosis than the patients in the control group, although this was not a statistically significant difference. No significant difference in functional bowel outcome was found between the early and late endosponge groups.

Limitations of this study are the small number of included patients and the long period over which patients were entered into our database, even though we have reported the fifth largest series worldwide. There might have been selection bias because of the retrospective design. Patients diagnosed with AL who were offered other therapies (i.e., dismantling of the anastomosis with creation of an end or loop colostomy) or transanal tube drainage were not included in our analysis. Patients with abdominal sepsis or generalized peritonitis were selected for dismantling of the anastomosis rather than endosponge therapy. Furthermore, no baseline LARS questionnaires were used.

Endosponge therapy resulted in a high rate of restored gastrointestinal continuity. Despite this positive outcome, a substantial number of patients had major LARS after an $\mathrm{AL}$ and endosponge therapy. This factor together with the duration of the therapy with several endosponge changes, frequent hospital visits, psychological factors and the occurrence of late abscess recurrence after successful endosponge therapy needs to be considered before the start of the endosponge therapy and could be of importance for the informed consent procedure. Future studies should evaluate which patients benefit from endosponge therapy over conventional treatment.

\section{Conclusions}

Our results show that endosponge therapy is an effective treatment for the closure of presacral cavities after rectal surgery with high success rate (88\%) leading to restored gastrointestinal continuity in $73 \%$ of patients. A significant proportion of patients developed major LARS despite endosponge treatment. This should be taken into consideration when contemplating salvaging an anastomosis with endosponge.

\section{Compliance with ethical standards}

Conflict of interest The authors declare that they have no conflict of interest.

Ethical approval The study was approved by the medical ethics committee of Isala hospital (reference number: 171215) and have been 
performed in accordance with the ethical standards as laid down in the 1964 Declaration of Helsinki.

Informed consent For this type of study, formal consent is not required.

\section{References}

1. Snijders HS, Wouters MW, van Leersum NJ, Kolfschoten NE, Henneman D, de Vries AC et al (2012) Meta-analysis of the risk for anastomotic leakage, the postoperative mortality caused by leakage in relation to the overall postoperative mortality. Eur J Surg Oncol 38(11):1013-1019

2. Gu WL, Wu SW (2015) Meta-analysis of defunctioning stoma in low anterior resection with total mesorectal excision for rectal cancer: evidence based on thirteen studies. World J Surg Oncol 13:9-014-0417-1

3. Qu H, Liu Y, Bi DS (2015) Clinical risk factors for anastomotic leakage after laparoscopic anterior resection for rectal cancer: a systematic review and meta-analysis. Surg Endosc 29(12):3608-3617

4. Wu SW, Ma CC, Yang Y (2014) Role of protective stoma in low anterior resection for rectal cancer: a meta-analysis. World J Gastroenterol 20(47):18031-18037

5. Borstlap WAA, Westerduin E, Aukema TS, Bemelman WA, Tanis PJ, Dutch snapshot research group (2017) anastomotic leakage and chronic presacral sinus formation after low anterior resection: results from a large cross-sectional study. Ann Surg 266(5):870-877

6. van Koperen PJ, van der Zaag ES, Omloo JM, Slors JF, Bemelman WA (2011) The persisting presacral sinus after anastomotic leakage following anterior resection or restorative proctocolectomy. Colorectal Dis 13(1):26-29

7. Weidenhagen R, Gruetzner KU, Wiecken T, Spelsberg F, Jauch KW (2008) Endoscopic vacuum-assisted closure of anastomotic leakage following anterior resection of the rectum: a new method. Surg Endosc 22(8):1818-1825

8. Nagell CF, Holte K (2006) Treatment of anastomotic leakage after rectal resection with transrectal vacuum-assisted drainage (VAC). A method for rapid control of pelvic sepsis and healing. Int $\mathbf{J}$ Colorectal Dis 21(7):657-660

9. Mees ST, Palmes D, Mennigen R, Senninger N, Haier J, Bruewer M (2008) Endo-vacuum assisted closure treatment for rectal anastomotic insufficiency. Dis Colon Rectum 51(4):404-410
10. van Koperen PJ, van Berge Henegouwen MI, Rosman C, Bakker CM, Heres P, Slors JF et al (2009) The Dutch multicenter experience of the endo-sponge treatment for anastomotic leakage after colorectal surgery. Surg Endosc 23(6):1379-1383

11. Strangio G, Zullo A, Ferrara EC, Anderloni A, Carlino A, Jovani $\mathrm{M}$ et al (2015) Endo-sponge therapy for management of anastomotic leakages after colorectal surgery: a case series and review of literature. Dig Liver Dis 47(6):465-469

12. Gardenbroek TJ, Musters GD, Buskens CJ, Ponsioen CY, D'Haens GR, Dijkgraaf MG et al (2015) Early reconstruction of the leaking ileal pouch-anal anastomosis: a novel solution to an old problem. Colorectal Dis 17(5):426-432

13. Borstlap WAA, Musters GD, Stassen LPS, van Westreenen HL, Hess D, van Dieren S et al (2018) Vacuum-assisted early transanal closure of leaking low colorectal anastomoses: the CLEAN study. Surg Endosc 32(1):315-327

14. Hupkens BJ, Breukink SO, Olde Reuver Of Briel C, Tanis PJ, de Noo ME, van Duijvendijk P et al. (2018) Dutch validation of the Low Anterior Resection Syndrome Score. Colorectal Dis 20:881-887

15. Emmertsen KJ, Laurberg S (2012) Low anterior resection syndrome score: development and validation of a symptom-based scoring system for bowel dysfunction after low anterior resection for rectal cancer. Ann Surg 255(5):922-928

16. Mussetto A, Arena R, Buzzi A, Fuccio L, Dari S, Brancaccio ML et al (2017) Long-term efficacy of vacuum-assisted therapy (Endo-SPONGE $((\mathrm{R}))$ ) in large anastomotic leakages following anterior rectal resection. Ann Gastroenterol 30(6):649-653

17. Riss S, Stift A, Kienbacher C, Dauser B, Haunold I, Kriwanek S et al (2010) Recurrent abscess after primary successful endosponge treatment of anastomotic leakage following rectal surgery. World J Gastroenterol 16(36):4570-4574

18. Sturiale A, Martellucci J, Zurli L, Vaccaro C, Brusciano L, Limongelli P et al (2017) Long-term functional follow-up after anterior rectal resection for cancer. Int J Colorectal Dis 32(1):83-88

19. Keane C, Wells C, O'Grady G, Bissett IP (2017) Defining low anterior resection syndrome: a systematic review of the literature. Colorectal Dis 19(8):713-722

Publisher's Note Springer Nature remains neutral with regard to jurisdictional claims in published maps and institutional affiliations. 